Celestial Mechanics and Dynamical Astronomy, Vol.94. No.1. (2006) pp.1-15.

\title{
Gravitational Field of Fractal Distribution of Particles
}

\author{
Vasily E. Tarasov \\ Skobeltsyn Institute of Nuclear Physics, \\ Moscow State University, Moscow 119992, Russia \\ E-mail: tarasov@theory.sinp.msu.ru
}

\begin{abstract}
In this paper we consider the gravitational field of fractal distribution of particles. To describe fractal distribution, we use the fractional integrals. The fractional integrals are considered as approximations of integrals on fractals. Using the fractional generalization of the Gauss's law, we consider the simple examples of the fields of homogeneous fractal distribution. The examples of gravitational moments for fractal distribution are considered.
\end{abstract}

PACS: 98.62.Ck; 05.45.Df; 45.50.-j; 98.80.Jk; 98.65.-r

Keywords: fractals, fractional integrals, gravitational multipole moment

\section{Introduction}

The aim of this paper is to consider the general properties of gravitational field that is generated by a fractal distribution of particles. This problem is nowadays particularly relevant. In fact, there is a general agreement that galaxy distribution exhibits fractal behavior up to a certain scale [1, 2]. The eventual presence of a transition scale towards homogeneity and the exact value of the fractal dimension are still matters of debate. Moreover it has been observed that cold gas clouds of the interstellar medium has a fractal structure, with $1.5 \leq D \leq 2$ in a large range of length scales [3, 4]. Derivatives and integrals of fractional order [5] have found many applications in recent studies of fractal structures. Fractional analysis can have numerous applications: kinetic theories 6. 7, 8, 11]; statistical mechanics [9, 10, 12]; dynamics in complex media [13, 14, 15, 16, 17]; 
and many others. The new type of problem has increased rapidly in areas in which the fractal features of a process or the medium impose the necessity of using non-traditional tools in "regular" smooth physical equations. In order to use fractional derivatives and fractional integrals for fractal distribution, we must use some continuous medium model [14, 16, 17].

We propose to describe the fractal distribution by a "fractional" continuous medium [14, where all characteristics and fields are defined everywhere in the volume but they follow some generalized equations which are derived by using fractional integrals. In many problems the real fractal structure of medium can be disregarded and the fractal distribution can be replaced by some "fractional" continuous mathematical model. Smoothing of microscopic characteristics over the physically infinitesimal volume transforms the initial fractal distribution into "fractional" continuous model 14 that uses the fractional integrals. The order of fractional integral is equal to the fractal dimension of distribution. The fractional integrals allow us to take into account the fractality of the distribution. Fractional integrals are considered as an approximation of integrals on fractals [18, 19. It was proved in [18] that integrals on net of fractals can be approximated by fractional integrals. In Ref. 9], we proved that fractional integrals can be considered as integrals over the space with fractional dimension up to numerical factor. In order to prove, we use the formulas of dimensional regularizations [20].

In this paper, we consider gravitational field of fractal distribution of particles. Fractal distribution is described by fractional continuous medium model [14, 16, 8, 17]. In the general case, the fractal distribution of particles cannot be considered as continuous medium. There are points and domains that are not filled of particles. In Ref. [14, we suggest to consider the fractal distributions as special ("fractional") continuous media. We use the procedure of replacement of the distribution with fractal mass dimension by some continuous model that uses fractional integrals. This procedure is a fractional generalization of Christensen approach [21]. Suggested procedure leads to the fractional integration and differentiation to describe fractal distribution.

In Section 2, the density of fractal distribution of mass is considered. In Section 3, we consider the simple examples of the gravitational field of homogeneous fractal distribution. In Section 4, the examples of gravitational quadrupole moments for fractal distribution are considered. Finally, a short conclusion is given in Section 5 . 


\section{Mass and Balance of Mass for Fractal Distribution}

\subsection{Mass of Fractal Distribution}

Let us consider a fractal distribution of particles. For example, we can assume that particles with a constant density are distributed over the fractal. In this case, the number of particles $N$ enclosed in a volume of characteristic size $R$ satisfies the scaling law

$$
N(R) \sim R^{D},
$$

whereas for a regular n-dimensional Euclidean object we have $N(R) \sim R^{n}$.

For distribution of particles with number density $n(\mathbf{r}, t)$, we have that the mass density can be defined by

$$
\rho(\mathbf{r}, t)=m n(\mathbf{r}, t),
$$

where $m$ is the mass of a particle. The total mass of region $W$ is then given by the integral

$$
M(W)=\int_{W} \rho(\mathbf{r}, t) d V_{3},
$$

or $M(W)=m N(W)$, where $N(W)$ is a number of particles in the region $W$. The fractional generalization of this equation can be written in the following form:

$$
M(W)=\int_{W} \rho_{D}(\mathbf{r}, t) d V_{D},
$$

where $D$ is a mass fractal dimension of the distribution, and $d V_{D}$ is an element of Ddimensional volume such that

$$
d V_{D}=C_{3}(D, \mathbf{r}) d V_{3} .
$$

For the Riesz definition of the fractional integral [5], the function $C_{3}(D, \mathbf{r})$ is defined by the relation

$$
C_{3}(D, \mathbf{r})=\frac{2^{3-D} \Gamma(3 / 2)}{\Gamma(D / 2)}|\mathbf{r}|^{D-3} .
$$

The initial points of the fractional integral are set to zero. The numerical factor in Eq. (66) has this form in order to derive usual integral in the limit $D \rightarrow(3-0)$. Note that the usual numerical factor $\gamma_{3}^{-1}(D)=\Gamma(1 / 2) / 2^{D} \pi^{3 / 2} \Gamma(D / 2)$, which is used in Ref. [5] leads to $\gamma_{3}^{-1}(3-0)=1 /\left[4 \pi^{3 / 2}\right]$ in the limit $D \rightarrow(3-0)$.

For the Riemann-Liouville fractional integral [5], the function $C_{3}(D, \mathbf{r})$ is defined by

$$
C_{3}(D, \mathbf{r})=\frac{|x y z|^{D / 3-1}}{\Gamma^{3}(D / 3)}
$$


Here we use Cartesian's coordinates $x, y$, and $z$. In order to have the usual dimensions of the physical values, we can use vector $\mathbf{r}$, and coordinates $x, y, z$ as dimensionless values. Therefore the density $\rho_{D}$ has the dimension of mass.

Note that the interpretation of fractional integration is connected with fractional dimension 9, 10. This interpretation follows from the well-known formulas for dimensional regularizations [20]. The fractional integral can be considered as an integral in the fractional dimension space up to the numerical factor $\Gamma(D / 2) /\left(2 \pi^{D / 2} \Gamma(D)\right)$.

If we consider the ball region $W=\{\mathbf{r}:|\mathbf{r}| \leq R\}$, and the spherically symmetric distribution of particles $\left(n_{D}(\mathbf{r}, t)=n_{D}(r)\right)$, then we have

$$
N(R)=4 \pi \frac{2^{3-D} \Gamma(3 / 2)}{\Gamma(D / 2)} \int_{0}^{R} n_{D}(r) r^{D-1} d r, \quad M(R)=m N(R) .
$$

For the homogeneous $\left(n_{D}(r)=n_{0}\right)$ fractal distribution, we get

$$
N(R)=4 \pi n_{0} \frac{2^{3-D} \Gamma(3 / 2)}{\Gamma(D / 2)} \frac{R^{D}}{D} \sim R^{D} .
$$

Fractal distribution of particles is called a homogeneous fractal distribution if the power law $N(R) \sim R^{D}$ does not depend on the translation of the region. The homogeneity property of the distribution can be formulated in the following form: for all regions $W$ and $W^{\prime}$ such that the volumes are equal $V(W)=V\left(W^{\prime}\right)$, we have that the number of particles in these regions are equal too, $N(W)=N\left(W^{\prime}\right)$. Note that the wide class of the fractal media satisfies the homogeneous property. In many cases, we can consider the porous media [23, 24], polymers [25], colloid aggregates [26], and aerogels [27] as homogeneous fractal media. In Refs. [14, 16], the continuous medium model for the fractal distribution was suggested. Note that the fractality and homogeneity properties can be realized for the fractional continuous model in the following forms:

(1) Homogeneity: the local number density of homogeneous fractal distribution is translation invariant value that has the form $n(\mathbf{r})=n_{0}=$ const.

(2) Fractality: the number of particles in the ball region $W$ obeys a power law relation $N_{D}(W) \sim R^{D}$, where $D<3$, and $R$ is the radius of the ball.

\subsection{Flow of Fractal Medium}

For distribution of particles with number density $n(\mathbf{r}, t)$ flowing with velocity $\mathbf{u}=\mathbf{u}(\mathbf{r}, t)$, the resulting density $\mathbf{J}(\mathbf{r}, t)$ is given by

$$
\mathbf{J}(\mathbf{r}, t)=m n(\mathbf{r}, t) \mathbf{u},
$$


where $m$ is the mass of a particle. Measuring the field $\mathbf{J}(\mathbf{r}, t)$ passing through a surface $S=\partial W$ gives the flow (flux of mass)

$$
I(S)=\Phi_{J}(S)=\int_{S}\left(\mathbf{J}, d \mathbf{S}_{2}\right)
$$

where $\mathbf{J}=\mathbf{J}(\mathbf{r}, t)$ is the flow field vector, $d \mathbf{S}_{\mathbf{2}}=d S_{2} \mathbf{n}$ is a differential unit of area pointing perpendicular to the surface $S$, and the vector $\mathbf{n}=n_{k} \mathbf{e}_{k}$ is a vector of normal. The fractional generalization of this equation for the fractal distribution can be written in the following form

$$
I(S)=\int_{S}\left(\mathbf{J}(\mathbf{r}, t), d \mathbf{S}_{d}\right)
$$

where we use

$$
d S_{d}=C_{2}(d, \mathbf{r}) d S_{2}, \quad C_{2}(d, \mathbf{r})=\frac{2^{2-d}}{\Gamma(d / 2)}|\mathbf{r}|^{d-2} .
$$

Note that $C_{2}(2, \mathbf{r})=1$ for $d=2$. In general, the medium on the boundary $\partial W$ has the

dimension $d$. In the general case, the dimension $d$ is not equal to 2 and is not equal to $(D-1)$.

\subsection{Equation of Continuity for Fractal Distribution}

The change of mass inside a region $W$ bounded by the surface $S=\partial W$ is always equal to the flux of mass through this surface. This is known as the law of mass conservation or the equation of balance of mass [16. If we denote by $\mathbf{J}(\mathbf{r}, t)$ the flow density, then mass conservation is written

$$
\frac{d M(W)}{d t}=-I(S)
$$

or, in the form

$$
\frac{d}{d t} \int_{W} \rho_{D}(\mathbf{r}, t) d V_{D}=-\oint_{\partial W}\left(\mathbf{J}(\mathbf{r}, t), d \mathbf{S}_{d}\right)
$$

In particular, when the surface $S=\partial W$ is fixed, we can write

$$
\frac{d}{d t} \int_{W} \rho_{D}(\mathbf{r}, t) d V_{D}=\int_{W} \frac{\partial \rho_{D}(\mathbf{r}, t)}{\partial t} d V_{D}
$$

Using the fractional generalization of the mathematical Gauss's theorem (see Appendix), we have

$$
\oint_{\partial W}\left(\mathbf{J}(\mathbf{r}, t), d \mathbf{S}_{d}\right)=\int_{W} C_{3}^{-1}(D, \mathbf{r}) \frac{\partial}{\partial x_{k}}\left(C_{2}(d, \mathbf{r}) J_{k}(\mathbf{r}, t)\right) d V_{D} .
$$

Substituting the right hand sides of Eqs. (16) and (17) in Eq. (15), we find the equation of balance of mass in differential form

$$
C_{3}(D, \mathbf{r}) \frac{\partial \rho_{D}(\mathbf{r}, t)}{\partial t}+\frac{\partial}{\partial x_{k}}\left(C_{2}(d, \mathbf{r}) J_{k}(\mathbf{r}, t)\right)=0 .
$$


This equation can be considered as a continuity equation for fractal distribution of particles [16].

\section{Gravitational Field of Fractal Distribution}

\subsection{Gravitational Field}

For a point mass $M$ at position $\mathbf{r}^{\prime}$ the gravitational field $\mathbf{F}$ at a point $\mathbf{r}$ is defined by

$$
\mathbf{F}=G M \frac{\mathbf{r}^{\prime}-\mathbf{r}}{\left|\mathbf{r}^{\prime}-\mathbf{r}\right|^{3}}
$$

where $G$ is a gravitational constant.

For a continuous distribution $\rho\left(\mathbf{r}^{\prime}\right)$ of mass, the gravitational field $\mathbf{F}$ at a point $\mathbf{r}$ is given by

$$
\mathbf{F}_{3}(\mathbf{r})=G \int_{W} \frac{\mathbf{r}^{\prime}-\mathbf{r}}{\left|\mathbf{r}^{\prime}-\mathbf{r}\right|^{3}} \rho\left(\mathbf{r}^{\prime}\right) d V_{3}^{\prime}
$$

The fractional generalization of this equation for a fractal distribution of mass is given by the equation

$$
\mathbf{F}_{D}(\mathbf{r})=G \int_{W} \frac{\mathbf{r}^{\prime}-\mathbf{r}}{\left|\mathbf{r}^{\prime}-\mathbf{r}\right|^{3}} \rho_{D}\left(\mathbf{r}^{\prime}\right) d V_{D}^{\prime},
$$

where $d V_{D}^{\prime}=C_{3}\left(D, \mathbf{r}^{\prime}\right) d V_{3}^{\prime}$. Eq. (21) can be considered as Newton's law written for a fractal distribution of particles.

Measuring the gravitational field passing through a surface $S=\partial W$ gives the gravitational filed flux

$$
\Phi_{F}(S)=\int_{S}\left(\mathbf{F}, d \mathbf{S}_{2}\right),
$$

where $\mathbf{F}$ is the gravitational field vector, and $d \mathbf{S}_{2}$ is a differential unit of area pointing perpendicular to the surface $S$.

\subsection{Gauss's Law for Fractal Distribution}

The Gauss's law tells us that the total flux $\Phi_{F}(S)$ of the gravitational field $\mathbf{F}$ through a closed surface $S=\partial W$ is proportional to the total mass $M(W)$ inside the surface:

$$
\Phi_{F}(\partial W)=4 \pi G M(W)
$$

For the fractal distribution, Gauss's law states

$$
\int_{S}\left(\mathbf{F}_{D}, d \mathbf{S}_{2}\right)=4 \pi G \int_{W} \rho_{D}(\mathbf{r}) d V_{D},
$$


where $\mathbf{F}=\mathbf{F}(\mathbf{r})$ is the gravitational field, and $\rho_{D}(\mathbf{r})$ is the mass density, $d V_{D}=C_{3}(D, \mathbf{r}) d V_{3}$, and $G$ is the gravitational constant.

Gauss's law by itself can be used to find the gravitational field of a point mass at rest, and the principle of superposition can then be used to find the gravitational field of an arbitrary fractal mass distribution.

If we consider the spherically symmetric fractal distribution $\rho_{D}(\mathbf{r})=\rho_{D}(r)$, and the ball region $W=\{\mathbf{r}:|\mathbf{r}| \leq R\}$, then we have

$$
M(W)=4 \pi \int_{0}^{R} \rho_{D}(r) C_{3}(D, \mathbf{r}) r^{2} d r
$$

where $C_{3}(D, \mathbf{r})$ is defined in Eq. (6), i.e.,

$$
M(W)=4 \pi \frac{2^{3-D} \Gamma(3 / 2)}{\Gamma(D / 2)} \int_{0}^{R} \rho_{D}(r) r^{D-1} d r .
$$

Using the sphere $S=\{\mathbf{r}:|\mathbf{r}|=R\}$ as a surface $S=\partial W$, we get

$$
\Phi_{F}(\partial W)=4 \pi R^{2} F_{D}(R)
$$

Substituting Eqs. (26) and (27) in the Gauss's law (23), we get the equation for gravitational field. As the result, the Gauss's law for fractal distribution with spherical symmetry leads us to the equation for gravitational field

$$
F_{D}(R)=\frac{\pi G 2^{5-D} \Gamma(3 / 2)}{R^{2} \Gamma(D / 2)} \int_{0}^{R} \rho_{D}(r) r^{D-1} d r .
$$

For example, the gravitational field of homogeneous $\left(\rho_{D}(\mathbf{r})=\rho_{\mathbf{0}}\right)$ spherically symmetric fractal distribution of mass is defined by

$$
F_{D}(R)=\rho_{0} \frac{\pi G 2^{5-D} \Gamma(3 / 2)}{D \Gamma(D / 2)} R^{D-2} \sim R^{D-2} .
$$

\section{Multipole Moments for Fractal Distribution}

\subsection{Multipole Expansion}

A multipole expansion is a series expansion of the effect produced by a given system in terms of an expansion parameter which becomes small as the distance away from the system increases. Therefore, the leading one of the terms in a multipole expansion are generally the strongest. The first-order behavior of the system at large distances can therefore be obtained from the first terms of this series, which is generally much easier 
to compute than the general solution. Multipole expansions are most commonly used in problems involving the gravitational field of mass aggregations, the gravity and magnetic fields of mass and flow distributions, and the propagation of electromagnetic waves.

To compute one particular case of a multipole expansion, let $\mathbf{R}=X_{k} \mathbf{e}_{k}$ be the vector from a fixed reference point to the observation point; $\mathbf{r}=x_{k} \mathbf{e}_{k}$ be the vector from the reference point to a point in the distribution; and $\mathbf{d}=\mathbf{R}-\mathbf{r}$ be the vector from a point in the distribution to the observation point. The law of cosines then yields

$$
d^{2}=R^{2}\left(1+\frac{r^{2}}{R^{2}}-2 \frac{r}{R} \cos \theta\right)
$$

where $d=|\mathbf{d}|$, and $\cos \theta=(\mathbf{r}, \mathbf{R}) /(r R)$, so

$$
d=R \sqrt{1+\frac{r^{2}}{R^{2}}-2 \frac{r}{R} \cos \theta}
$$

Now define $\epsilon=r / R$, and $x=\cos \theta$, then

$$
\frac{1}{d}=\frac{1}{R}\left(1-2 \epsilon x+\epsilon^{2}\right)^{-1 / 2}
$$

But $\left(1-2 \epsilon x+\epsilon^{2}\right)^{-1 / 2}$ is the generating function for Legendre polynomials $P_{n}(x)$ as follows:

$$
\left(1-2 \epsilon x+\epsilon^{2}\right)^{-1 / 2}=\sum_{n=0}^{\infty} \epsilon^{n} P_{n}(x)
$$

so, we have the equation

$$
\frac{1}{d}=\frac{1}{R} \sum_{n=0}^{\infty}\left(\frac{r}{R}\right)^{n} P_{n}(\cos \theta)
$$

The gravitational potential $U(\mathbf{F}=-\nabla U)$ obeys $(1 / d)$ law. Therefore, this potential can be expressed as a multipole expansion

$$
U=-G \sum_{n=0}^{\infty} \frac{1}{R^{n+1}} \int_{W} r^{n} P_{n}(\cos \theta) \rho_{D}(\mathbf{r}) d V_{D}
$$

The $n=0$ term of this expansion can be pulled out by noting that $P_{0}(x)=1$, so

$$
U=-\frac{G}{R} \int_{W} \rho_{D}(\mathbf{r}) d V_{D}-G \sum_{n=1}^{\infty} \frac{1}{R^{n+1}} \int_{W} r^{n} P_{n}(\cos \theta) \rho_{D}(\mathbf{r}) d V_{D}
$$

The nth term

$$
U_{n}=-\frac{G}{R^{n+1}} \int_{W} r^{n} P_{n}(\cos \theta) \rho_{D}(\mathbf{r}) d V_{D}
$$

is commonly named multipole, and for $n=2$ - quadrupole. 


\subsection{Gravitational Moment of Fractal Distribution}

Gravitational moments describe the nonuniform distribution of mass. The gravitational quadrupole term is given by

$$
\begin{aligned}
& U_{2}=-\frac{G}{R^{3}} \int_{W} r^{2} P_{2}(\cos \theta) \rho_{D}(\mathbf{r}) d V_{D}= \\
& =-\frac{G}{2 R^{3}} \int_{W} r^{2}\left(3 \cos ^{2} \theta-1\right) \rho_{D}(\mathbf{r}) d V_{D}= \\
& =-\frac{G}{2 R^{3}} \int_{W}\left(\frac{3}{R^{2}}(\mathbf{R}, \mathbf{r})^{2}-r^{2}\right) \rho_{D}(\mathbf{r}) d V_{D}
\end{aligned}
$$

The quadrupole is the third term in a gravitational multipole expansion, and can be defined by

$$
U_{2}=-\frac{G}{2 R^{3}} \sum_{k, l=1}^{3} \frac{X_{k} X_{l}}{R^{2}} M_{k l},
$$

where $G$ is the gravitational constant, $R$ is the distance from the fractal distribution of mass, and $M_{k l}$ is the gravitational quadrupole moment, which is a tensor.

The gravitational quadrupole moment is defined by the equation

$$
M_{k l}=\int_{W}\left(3 x_{k} x_{l}-r^{2} \delta_{k l}\right) \rho_{D}(\mathbf{r}) d V_{D},
$$

where $x_{k}=x, y$, or $z$. From this definition, it follows that

$$
M_{k l}=M_{l k}, \quad \text { and } \quad \sum_{k=1}^{3} M_{k k}=0 .
$$

Therefore, we have $M_{z z}=-M_{x x}-M_{y y}$. In order to compute the values

$$
\begin{gathered}
M_{x x}^{(D)}=\int_{W}\left[2 x^{2}-y^{2}-z^{2}\right] \rho_{D}(\mathbf{r}) d V_{D}, \\
\left.M_{y y}^{(D)}=\int_{W}\left[-x^{2}+2 y^{2}-z^{2}\right)\right] \rho_{D}(\mathbf{r}) d V_{D}, \\
\left.M_{z z}^{(D)}=\int_{W}\left[-x^{2}-y^{2}+2 z^{2}\right)\right] \rho_{D}(\mathbf{r}) d V_{D},
\end{gathered}
$$

we consider the following expression

$$
\left.M(\alpha, \beta, \gamma)=\int_{W}\left[\alpha x^{2}+\beta y^{2}+\gamma z^{2}\right)\right] \rho_{D}(\mathbf{r}) d V_{D}
$$


where we use the Riemann-Liouville fractional integral [5], and the function $C_{3}(D, \mathbf{r})$ in the form

$$
C_{3}(D, \mathbf{r})=\frac{|x y z|^{a-1}}{\Gamma^{3}(a)}, \quad a=D / 3
$$

Using Eq. (47), we have

$$
M_{x x}^{(D)}=M(2,-1,-1), \quad M_{x x}^{(D)}=M(-1,2,-1), \quad M_{z z}^{(D)}=M(-1,-1,2) .
$$

\subsection{Quadrupole Moment of Fractal Parallelepiped}

Let us consider the example of gravitational quadrupole moment for the homogeneous $\left(\rho_{D}(\mathbf{r})=\rho_{0}\right)$ fractal distribution in the parallelepiped region

$$
W=\{(x ; y ; z): 0 \leq x \leq A, 0 \leq y \leq B, 0 \leq z \leq C\} .
$$

If we consider the region $W$ in the form (50), then we get (47) in the form

$$
M(\alpha, \beta, \gamma)=\frac{\rho_{0}(A B C)^{a}}{(a+2) a^{2} \Gamma^{3}(a)}\left(\alpha A^{2}+\beta B^{2}+\gamma C^{2}\right) .
$$

The total mass of this region $W$ is

$$
M(W)=\rho_{0} \int_{W} d V_{D}=\frac{\rho_{0}(A B C)^{a}}{a^{3} \Gamma^{3}(a)} .
$$

Therefore, we have the following equation

$$
M(\alpha, \beta, \gamma)=\frac{a}{a+2} M(W)\left(\alpha A^{2}+\beta B^{2}+\gamma C^{2}\right),
$$

where $a=D / 3$. If $D=3$, then we have

$$
M(\alpha, \beta, \gamma)=\frac{1}{3} M(W)\left(\alpha A^{2}+\beta B^{2}+\gamma C^{2}\right)
$$

As the result, we get gravitational quadrupole moments $M_{k k}^{(D)}$ of fractal distribution in the region $W$ :

$$
M_{k k}^{(D)}=\frac{3 D}{D+6} M_{k k}^{(3)}
$$

where $M_{k k}^{(3)}$ are moments for the usual homogeneous distribution $(D=3)$. By analogy with these equations, we can derive $M_{k l}^{(D)}$ for the case $k \neq l$. These quadrupole moments are

$$
M_{k l}^{(D)}=\frac{4 D^{2}}{(D+3)^{2}} M_{k l}^{(3)}, \quad(k \neq l) .
$$

Using $2<D \leq 3$, we get the relations

$$
0.75<\frac{3 D}{D+6} \leq 1, \quad 0.64<\frac{4 D^{2}}{(D+3)^{2}} \leq 1
$$

Quadrupole moment of fractal ellipsoid is considered in Appendix. 


\section{Conclusion}

The fractional continuous models for fractal distribution of particles can have a wide application. This is due in part to the relatively small numbers of parameters that define a random fractal distribution of great complexity and rich structure. In many cases, the real fractal structure of matter can be disregarded and the distribution of particles can be replaced by some fractional continuous model [14, 16]. In order to describe the distribution with non-integer dimension, we must use the fractional calculus. Smoothing of the microscopic characteristics over the physically infinitesimal volume transforms the initial fractal distribution into fractional continuous model that uses the fractional integrals. The order of fractional integral is equal to the fractal dimension of the distribution. The fractional continuous model for the fractal distribution allows us to describe dynamics of a wide class of fractal media [16, 17, 8].

The suggested results can have a wide application to galactic dynamics and cosmology. In particular, there is strong evidence that the distribution of mass beyond the scale of clusters of galaxies is fractal, with $D \simeq 1.2$, corresponding to a power-law two-point correlation function with exponent equal to -1.8 . Fractal distributions may also be present within gravitational systems of a smaller scale, for example, galaxies. However, the results are incomplete in the following aspect: it is known that the fractal distribution of mass in the Universe is characterized by large density fluctuations, even within the fractal volume, therefore it is far from homogeneous. The nonhomogeneity of the fractal distribution can be described by the suggested fractional continuous model [14]. The fluctuation deviation from homogeneity can be parametrized by the so-called n-point correlation functions, with $n>2$ [28, 29, 30, 31]. Such density fluctuations are important in that they produce terms in the force field which can be described only statistically. An elementary example is the random distribution of particles in a three-dimensional sphere. Although the distribution can be considered as uniform when viewed at the scale of the sphere (with $D=3$ ), the Poisson noise of the density field will cause gravitational clustering in small scales that will finally prevail the overall evolution of the system, i.e., the latter will be quite a different from the evolution of a perfectly homogeneous sphere. 


\section{Appendix}

\section{Fractional Gauss's theorem}

In order to realize the representation, we derive the fractional generalization of the Gauss's theorem

$$
\int_{\partial W}\left(\mathbf{J}(\mathbf{r}, t), d \mathbf{S}_{2}\right)=\int_{W} \operatorname{div}(\mathbf{J}(\mathbf{r}, t)) d V_{3},
$$

where the vector $\mathbf{J}(\mathbf{r}, t)=J_{k} \mathbf{e}_{k}$ is a field, and

$$
\operatorname{div}(\mathbf{J})=\frac{\partial \mathbf{J}}{\partial \mathbf{r}}=\frac{\partial J_{k}}{\partial x_{k}}
$$

Here and later we mean the sum on the repeated index $k$ from 1 to 3. Using Eq. (13), we get

$$
\int_{\partial W}\left(\mathbf{J}(\mathbf{r}, t), d \mathbf{S}_{d}\right)=\int_{\partial W} C_{2}(d, \mathbf{r})\left(\mathbf{J}(\mathbf{r}, t), d \mathbf{S}_{2}\right) .
$$

Note that we have $C_{2}(2, \mathbf{r})=1$ for the $d=2$. Using the usual Gauss's theorem (58), we get

$$
\int_{\partial W} C_{2}(d, \mathbf{r})\left(\mathbf{J}(\mathbf{r}, t), d \mathbf{S}_{2}\right)=\int_{W} \operatorname{div}\left(C_{2}(d, \mathbf{r}) \mathbf{J}(\mathbf{r}, t)\right) d V_{3} .
$$

Equations (5) and (6) in the form $d V_{3}=C_{3}^{-1}(D, \mathbf{r}) d V_{D}$ allows us to derive the fractional generalization of the Gauss's theorem:

$$
\int_{\partial W}\left(\mathbf{J}(\mathbf{r}, t), d \mathbf{S}_{d}\right)=\int_{W} C_{3}^{-1}(D, \mathbf{r}) \operatorname{div}\left(C_{2}(d, \mathbf{r}) \mathbf{J}(\mathbf{r}, t)\right) d V_{D} .
$$

\section{Quadrupole Moment of Fractal Ellipsoid}

Let us consider the example of gravitational quadrupole moment for the homogeneous $\left(\rho_{D}(\mathbf{r})=\rho_{0}\right)$ fractal distribution in the ellipsoid region $W$ :

$$
\frac{x^{2}}{A^{2}}+\frac{y^{2}}{B^{2}}+\frac{z^{2}}{C^{2}} \leq 1
$$

If we consider the region $W$ in the form (63), then we get (47) in the form

$$
M(\alpha, \beta, \gamma)=\frac{\rho_{0}(A B C)^{a}}{(3 a+2) \Gamma^{3}(a)}\left(\alpha A^{2} K_{1}(a)+\beta B^{2} K_{2}(a)+\gamma C^{2} K_{3}(a)\right)
$$

where $a=D / 3$, and $K_{i}(a)(\mathrm{i}=1,2,3)$ are defined by

$$
K_{1}(a)=L(a+1, a-1,2 \pi) L(a-1,2 a+1, \pi)
$$




$$
\begin{aligned}
& K_{2}(a)=L(a-1, a+1,2 \pi) L(a-1,2 a+1, \pi), \\
& K_{3}(a)=L(a-1, a-1,2 \pi) L(a+1,2 a-1, \pi) .
\end{aligned}
$$

Here we use the following function

$$
L(n, m, l)=\frac{2 l}{\pi} \int_{0}^{\pi / 2} d x|\cos (x)|^{n}|\sin (x)|^{m}=\frac{l}{\pi} \frac{\Gamma(n / 2+1 / 2) \Gamma(m / 2+1 / 2)}{\Gamma(n / 2+m / 2+1)} .
$$

If $D=3$, we obtain

$$
M(\alpha, \beta, \gamma)=\frac{4 \pi}{3} \frac{\rho_{0} A B C}{5}\left(\alpha A^{2}+\beta B^{2}+\gamma C^{2}\right)
$$

where we use $K_{1}=K_{2}=K_{3}=4 \pi / 3$. The total mass of this region $W$ is

$$
M(W)=\rho_{0} \int_{W} d V_{D}=\frac{\rho_{0}(A B C)^{a}}{3 a \Gamma^{3}(a)} \frac{2 \Gamma^{3}(a / 2)}{\Gamma(3 a / 2)} .
$$

If $D=3$, we have the total mass

$$
M(W)=\rho_{0} \int_{W} d V_{3}=\frac{4 \pi}{3} \rho_{0} A B C .
$$

Using Eqs. (69) and (170), we get the quadrupole moments (49) for fractal ellipsoid

$$
M(\alpha, \beta, \gamma)=\frac{a M(W)}{3 a+2}\left(\alpha A^{2}+\beta B^{2}+\gamma C^{2}\right)
$$

where $a=D / 3$. If $D=3$, then we have the well-known relation

$$
M(\alpha, \beta, \gamma)=\frac{M(W)}{5}\left(\alpha A^{2}+\beta B^{2}+\gamma C^{2}\right)
$$

\section{References}

[1] F. Sylos Labini, M. Montuori, and L. Pietronero, Phys. Rep. 293 (1998) 61-226.

[2] P.H. Coleman, and L. Pietronero, Phys. Rep. 213 (1992) 311-389.

[3] R.B. Larson, Month. Not. RAS. 194 (1981) 809.

[4] J.M. Scalo, in Interstellar Processes. Eds. D.J. Hollenbach, and H.A. Thronson, (Dordrecht: D. Reidel, 1987) pp.349-392.

[5] S.G. Samko, A.A. Kilbas and O.I. Marichev, Fractional Integrals and Derivatives Theory and Applications (Gordon and Breach, New York, 1993)

[6] G.M. Zaslavsky, Phys. Rep. 371 (2002) 461-580. 
[7] G.M. Zaslavsky, Physics of Chaos in Hamiltonian Dynamics, (Imperial College Press, London, 1998)

[8] V.E. Tarasov, and G.M. Zaslavsky, Physica A 354 (2005) 249-261; E-print physics/0511144.

[9] V.E. Tarasov, Chaos 14 (2004) 123-127; E-print nlin.CD/0312044.

[10] V.E. Tarasov, Phys. Rev. E, 71 (2005) 011102; E-print cond-mat/0505720.

[11] V.E. Tarasov, J. Phys. A 38 (2005) 5929-5943; E-print math.DS/0602208.

[12] V.E. Tarasov J. Phys. Conf. Ser. 7 (2005) 17-33; E-print nlin.CD/0602062

[13] R. Nigmatullin, Phys. Stat. Solidi B 133 (1986) 425-430.

[14] V.E. Tarasov, Phys. Lett. A 336 (2005) 167-174; E-print cont-mat/0506137.

[15] V.E. Tarasov, Phys. Lett. A 341 (2005) 467-472; E-print physics/0602121.

[16] V.E. Tarasov, Ann. Phys. 318 (2005) 286-307; E-print physics/0602096.

[17] V.E. Tarasov, Chaos 15 (2005) 023102; E-print nlin.CD/0602029.

[18] F.Y. Ren, J.R. Liang, X.T. Wang, and W.Y. Miu, Chaos, Solitons and Fractals 16 (2003) 107-117.

[19] A. Le Mehaute, R.R. Nigmatullin, L. Nivanen, Fleches du temps et geometric fractale (Hermes, Paris, 1998) Chapter 5.

[20] J.C. Collins, Renormalization (Cambridge University Press, Cambridge, 1984) Sec. 4.1.

[21] R.M. Christensen, Mechanics of Composite Materials (Wiley, New York, 1979)

[22] J. Feder, Fractals (Plenum Press, New York, 1988)

[23] P. Pfeifer, and D. Avnir, J. Chem. Phys. 79 (1983) 3558-3565, 3566-3571.

[24] H.D. Bale, and P.W. Schmidt, Phys. Rev. Lett. 53 (1984) 596-599.

[25] D.W. Schaefer, and K.D. Keefer, Phys. Rev. Lett. 53 (1984) 1383-1386.

[26] D.W. Schaefer, J.E. Martin, P. Wiltzius, and D.S. Cannell, Phys. Rev. Lett. 52 (1994) 2371-2374.

[27] Aerogels Ed. J. Fricke (Springer, Berlin, 1985)

[28] A. Gabrielli, F. Sylos Labini, and S. Pellegrini, Europhys. Lett. 46 (1999) 127-133; E-print astro-ph/9809234 and references therein.

[29] L. Pietronero, A. Gabrielli, and F. Sylos Labini, Physica A 306 (2002) 395-401; E-print astro-ph/0110169.

[30] F. Sylos Labini, and A. Gabrielli, Physica A 338 (2004) 44-49; E-print astro-ph/0401204

[31] A. Gabrielli, F. Sylos Labini, M. Joyce, and L. Pietronero, Statistical Physics of Cosmic Structures (Springer, New York, 2004) 